\title{
Optimal Sequences, Power Control, and User Capacity of Synchronous CDMA Systems with Linear MMSE Multiuser Receivers
}

\author{
Pramod Viswanath, Student Member, IEEE, Venkat Anantharam, Fellow, IEEE, \\ and David N. C. Tse, Member, IEEE
}

\begin{abstract}
There has been intense effort in the past decade to develop multiuser receiver structures which mitigate interference between users in spread-spectrum systems. While much of this research is performed at the physical layer, the appropriate power control and choice of signature sequences in conjunction with multiuser receivers and the resulting network user capacity is not well understood. In this paper we will focus on a single cell and consider both the uplink and downlink scenarios and assume a synchronous CDMA (S-CDMA) system. We characterize the user capacity of a single cell with the optimal linear receiver (MMSE receiver). The user capacity of the system is the maximum number of users per unit processing gain admissible in the system such that each user has its quality-of-service $(Q 0 S)$ requirement (expressed in terms of its desired signal-to-interference ratio) met. Our characterization allows us to describe the user capacity through a simple effective bandwidth characterization: Users are allowed in the system if and only if the sum of their effective bandwidths is less than the processing gain of the system. The effective bandwidth of each user is a simple monotonic function of its QoS requirement. We identify the optimal signature sequences and power control strategies so that the users meet their QoS requirement. The optimality is in the sense of minimizing the sum of allocated powers. It turns out that with this optimal allocation of signature sequences and powers, the linear MMSE receiver is just the corresponding matched filter for each user. We also characterize the effect of transmit power constraints on the user capacity.
\end{abstract}

Index Terms-CDMA, linear MMSE receivers, power control, user capacity, WBE sequences.

\section{INTRODUCTION}

A central problem in the design of wireless networks is how to use the limited resources such as bandwidth and power most efficiently in order to meet the quality-of-service requirements of applications in terms of bit rate and loss. To meet these challenges, there have been intense effort to develop more sophisticated physical layer communication techniques.

Manuscript received February 1, 1998; revised April 11, 1999. The work of P. Viswanath and V. Anantharam was supported by the National Science Foundation under Grants NCR 94-22513 and IRI 95-05561. The work of D. N. C. Tse was supported by the Air Force Office of Scientific Research under Grant F49620-96-1-0199, and by an NSF CAREER Award under Grant NCR-9734090. The material in this paper was presented in part at the IEEE International Symposium on Information Theory, MIT, Cambridge, MA, August 16-21, 1998.

The authors are with the Electrical Engineering and Computer Science Department, University of California at Berkeley, Berkeley, CA 94720 USA (e-mail: \{pvi, ananth, dtse $\} @$ eecs.berkeley.edu).

Communicated by M. L. Honig, Associate Editor for Communications.

Publisher Item Identifier S 0018-9448(99)05880-0.
A significant thrust of work has been on developing multiuser receiver structures which mitigate the interference between users in spread-spectrum systems. (See, for example, [21], [8], [9], [26], [10], [14], and [15].)

In this paper, we focus on a synchronous power-controlled CDMA system with multiuser receivers. The processing gain represents the degrees of freedom in the system. We assume that at the receiver, each user is demodulated using a linear receiver structure; in particular, we shall focus on the "linear minimum mean-square error (MMSE) receiver" (formally defined in Section II; also see [10]). We are interested in the user capacity of both the uplink (mobiles to base station) and the downlink (base station to mobiles) of this system equipped with linear MMSE multiuser receiver. We say that a set of users is admissible in the uplink system with processing gain $N$ if one can allot signature sequences to the users and control their received power such that the achieved signalto-interference (SIR) of each user is greater than its SIR requirement. We are interested in the problem of characterizing the maximum number of users per degree of freedom, called the user capacity of the uplink system. Analogous definitions of admissibility and user capacity can be made for the downlink. For the most part, in this paper we shall focus on the uplink (the downlink scenario turns out to be very similar and we briefly summarize the results) and our main results are as follows.

1) $K$ users with SIR requirements $\beta_{1}, \cdots, \beta_{K}$ are admissible in the system with processing gain $N$ if and only if

$$
\sum_{i=1}^{K} \frac{\beta_{i}}{1+\beta_{i}}<N .
$$

This allows us to characterize the admissibility of users via a notion of effective bandwidth. If we consider $\frac{\beta}{1+\beta}$ as the effective bandwidth of a user with SIR requirement $\beta$, then users are admissible if and only if the sum of their effective bandwidths is less than the processing gain of the system.

2) Our proof of the admissibility of users with SIR requirements $\beta_{1}, \cdots, \beta_{K}$ is constructive in nature, i.e., we explicitly allocate powers and signature sequences so that users' SIR requirements are met (such allocations are called valid). Among the class of valid allocations, 
we identify the optimal allocation, optimal in the sense of minimizing the sum of allocated powers. The optimal allocation has the following structure.

a) A user is said to be oversized if the user's effective bandwidth is large relative to the effective bandwidths of the other users. Oversized users are allocated orthogonal signature sequences (hence independent channels) and powers proportional to their SIR requirements.

b) Nonoversized users are allocated sequences that we denote generalized Welch-bound-equality (WBE) sequences. These users are allocated powers proportional to their effective bandwidth.

3) With this allocation of signature sequences and powers, the MMSE linear receiver simplifies (somewhat unexpectedly) to the matched filter for each user. Thus the user capacity of a system using the a priori inferior matched filter receiver is the same as that of the system using MMSE linear receiver.

4) In the special case when the SIR requirements of all the users are equal (to, say, $\beta$ ), our main result simplifies to the following: $K$ users are admissible in the system with processing gain $N$ if and only if

$$
\frac{K}{N}<1+\frac{1}{\beta}
$$

Using the optimality (in the sense of minimizing sum of allocated powers) of the allocation scheme, we identify the precise loss in the admissibility of number of users as a function of the background noise power and the average received power constraint.

In [17], the authors consider the scenario when the signature sequences of the users are independent and randomly chosen. They show that the SIR of the users of a large system (with a large number of users and large processing gain) converges (in probability) and analyze the user capacity of the system based on the value to which the SIR converges. It is interesting to compare the performance of that system with the one considered here when the sequences are optimally chosen.

1) Under the MMSE receiver, the user capacity of a system using random sequences is asymptotically identical to that of a system with optimally chosen sequences. This holds when there are no transmit power constraints, or equivalently, when the background noise power is low. We will provide an explanation for this phenomenon.

2) Under the conventional matched-filter receiver, a system using random sequences admits one user per degree of freedom less than when the sequences are optimally chosen. This shows that while the MMSE and the matched-filter receivers have the same performance when the sequences are optimally chosen, the MMSE receiver is much more robust to the choice of spreading sequences.

3) Under transmit power constraints, systems employing random sequences admit strictly fewer users than the corresponding systems with optimal sequences. We quantify precisely the gap in performance.

In related work, there has been a great deal of research studying the problem of power control of the users for conventional CDMA systems. Distributed iterative algorithms that achieve power control of the users are discussed in, for example, [4] and [6]. These ideas were extended subsequently to systems with MMSE receivers [18], [7], but they focused on deriving convergent power control algorithms rather than analyzing the achievable user capacity. The problem of identifying good signature sequences has been studied in [12] in the context of a spread-spectrum system with conventional matched filter receiver and equal received power for all users. In [13], for equal received powers, the problem addressed in an information-theoretic setting is to identify signature sequences for which the sum capacity, the sum of rates of all the users at which reliable communication can take place, is maximized. To achieve sum capacity joint processing of the users is required (for example, MMSE receivers with successive cancellation; see [20]) while in this paper we restrict ourselves to single-user demodulators. Though the problem addressed in [13] is thus different from the problem addressed in this paper, the optimal sequences turn out to be identical. This statement is true even for the situation of unequal received powers, as shown in recent work [23].

An important special case subsumed by our framework is when the signature sequences are constrained to be chosen from an orthogonal sequence set. This corresponds to dividing the entire bandwidth into frequency slots (or channels), i.e., a joint FDMA/CDMA system. In this case, the receiver is trivial and both MMSE and matched-filter receiver structures coincide. Our main result in this framework is as follows: $K$ users each with SIR requirement $\beta$ are admissible in the system with processing gain $N$ if and only if $K<N\left\lfloor 1+\frac{1}{\beta}\right\rfloor$. We observe that the maximum number of users admissible per unit processing gain differs from the earlier results by an integer part. Thus we identify the gain by using nonorthogonal codes and multiuser linear receivers; the difference depends on the factor $\frac{1}{\beta}$ and the processing gain $N$. In the scenario when users are differentiated by their SIR requirement, we identify the user capacity of the system.

This paper is organized as follows: In Section II, we give a precise definition of the uplink model and of the admissibility of the users. User capacity of the uplink system with linear MMSE receivers for the situation of equal SIR requirements of the users is identified in Section III. In a physical system, the power transmitted by a user is constrained naturally. In Section IV, we demonstrate the optimality of a particular allocation scheme (developed in Section III) in the sense of minimizing the sum of allocated powers. This allows us to precisely quantify the loss in user capacity of the system with a received power constraint. Section V completely generalizes the results of Sections III and IV to the situation when users have different SIR requirements. In Section VI, we will focus on the downlink. We can ask our admissibility and user capacity region questions in this setup too. As can be expected, there is a lot of connection between the downlink and uplink scenarios 
and we summarize the results. Section VII focuses on the joint FDMA/CDMA setup that corresponds to the restriction of signature sequences to be chosen from an orthogonal sequence set and identifies the user capacity of the system under various settings. Section VIII contains our conclusions and discusses directions toward future work.

\section{Model AND DEFInitions}

\section{A. Standard Synchronous CDMA Model}

We consider a symbol-synchronous code-division multipleaccess (CDMA) system and focus on the uplink. Following the standard notation (see [22, Sec. 2.1]), the received signal in one symbol interval (of $T$ time units) can be expressed as

$$
y(t)=\sum_{i=1}^{K} X_{i} s_{i}(t)+n(t), \quad t \in[0, T] .
$$

Here $T$ is the inverse of the data rate, $K$ is the number of users, and $\left(s_{i}(t), t \in[0, T]\right)$ is the signature waveform of user $i$, and is thought of as an element of $L^{2}[0, T]$, the Hilbert space of square integrable functions on $[0, T]$. The waveform $\left(s_{i}(t), t \in[0, T]\right)$ is assumed to have unit norm. The information transmitted by each user is modeled as zero-mean, independent random variables $X_{1}, X_{2}, \cdots, X_{K}$. The variance $E\left[X_{i}^{2}\right]$ is the power at which user $i$ is received. We denote the received power of user $i$ as $p_{i}$, the product of the transmit power of user $i$ and the path gain from user $i$ to the receiver (base station). By the assumption of perfect power control or equivalently perfect channel estimation, we shall assume that we can allocate received powers for the users. The process $n(t)$ is additive white Gaussian noise independent of the user symbols $X_{1}, \cdots, X_{K}$.

Let the processing gain of the system be $N$. Following the usual notation (as in [22, Sec. 2.3.6]), the signature waveforms can then be written as

$$
s_{i}(t)=\sum_{j=1}^{N} s_{i}(j) \psi_{j}(t), \quad t \in[0, T]
$$

where $\left\{\left(\psi_{j}(t), t \in[0, T]\right)\right\}_{j=1} \cdots N$ is an orthonormal set in $L^{2}[0, T]$ and $s_{i}(j)$ is the inner product between the waveform of user $i$, namely, $\left(s_{i}(t), t \in[0 . T]\right)$ and $\left(\psi_{j}(t), t \in[0, T]\right)$. With some abuse of notation, let us represent the vector of inner products $\left(s_{i}(1), \cdots, s_{i}(N)\right)$ as $s_{i}$ the signature sequence of user $i$ ( $s_{i}$ is a vector in $\mathbb{S}_{1}^{N-1}$, the unit sphere in $\mathbb{R}^{N}$ ). It is well known that the projections $\left\{y_{j}\right\}_{j=1} \cdots N$ of $(y(t), t \in[0, T])$ on $\left\{\left(\psi_{j}(t), t \in\right.\right.$ $[0, T])\}_{j=1 \ldots N}$ are sufficient statistics (see [22, Sec. 2.9.2 and Ch. 3]) for the problem of demodulating the user symbols. Writing $Y=\left(y_{1}, \cdots, y_{N}\right)$ as a vector in $\mathbb{R}^{N}$, the received signal can be written as

$$
Y=\sum_{i=1}^{K} s_{i} X_{i}+W
$$

where $W$ is an independent and identically distributed (i.i.d.) Gaussian vector with covariance $\sigma^{2} I$, independent of the transmitted symbols. Henceforth, we consider the allocation of signature sequences for the users. By a suitable choice of the orthonormal waveform set $\left\{\left(\psi_{j}(t), t \in[0, T]\right)\right\}_{j=1 \cdots N}$, the signature waveforms for the users can be constructed (following (1)).

Suppose the symbol of user $i$ is decoded using a linear receiver, denoted by $c_{i}$ (a vector in $\mathbb{R}^{N}$ ), then the signal-tointerference ratio of user $i\left(\operatorname{SIR}_{i}\right)$ is

$$
\mathrm{SIR}_{i}=\frac{\left(c_{i}, s_{i}\right)^{2} p_{i}}{\sigma^{2}\left(c_{i}, c_{i}\right)+\sum_{j \neq i}\left(c_{i}, s_{j}\right)^{2} p_{j}} .
$$

We say that $K$ users are admissible in the system if there is an allocation of positive powers $p_{1}, \cdots, p_{K}$, signature sequences $s_{1}, \cdots, s_{K} \in \mathbb{S}_{1}^{N-1}$ and linear receiver structures $c_{1}, \cdots, c_{K}$ such that

$$
\operatorname{SIR}_{i} \geq \beta, \quad \forall i=1 \cdots K .
$$

Here $\beta>0$ is some fixed SIR requirement of each user that has to be met for satisfactory performance. Such a choice of powers and signature sequences is called a valid allocation.

\section{B. Structure of Optimum Linear Receiver}

It is well known that the MMSE receiver is the optimum linear receiver, optimum in the sense of maximizing the SIR of each user. While there are many derivations of the structure of the MMSE receiver (see [10], [17], [18] for example and [22, Sec. 6.2]) $c_{1}, \cdots, c_{K}$, we give an elementary derivation of the same as the argument of a problem of minimizing a convex function over a convex set below (this will also aid us in developing notation to be used in the characterization of the user capacity regions).

Fix the user powers $p_{1}, \cdots, p_{K}$, and the signature sequences $s_{1}, \cdots, s_{K}$. The optimum receiver $c_{i}$ is one that maximizes $\mathrm{SIR}_{i}$. Now, let

$$
\begin{aligned}
S & =\left[s_{1}, s_{2}, \cdots, s_{K}\right] \\
D & =\operatorname{diag}\left(p_{1}, \cdots, p_{K}\right)
\end{aligned}
$$

and

$$
\begin{aligned}
S_{i} & =\left[s_{1} \cdots, s_{i-1}, s_{i+1} \cdots, s_{K}\right] \\
D_{i} & =\operatorname{diag}\left(p_{1}, \cdots, p_{i-1}, p_{i+1} \cdots, p_{K}\right) .
\end{aligned}
$$

Let

$$
Z_{i}=S_{i} D_{i} S_{i}^{t}+\sigma^{2} I
$$

and

$$
Z=S D S^{t}+\sigma^{2} I
$$

and we note that they are positive definite. Let $Z_{i}=U_{i} \Lambda_{i} U_{i}^{t}$ for a positive diagonal matrix $\Lambda_{i}$ and unitary $U_{i}$. Also, let $S D S^{t}=U \Lambda U^{t}$. Then

$$
\begin{aligned}
& \max _{c_{i} \neq 0} \operatorname{SIR}_{i}=\max _{c_{i} \neq 0} \frac{\left(c_{i}, s_{i}\right)^{2} p_{i}}{c_{i}^{t} Z_{i} c_{i}} \\
&=p_{i} \max _{x_{i} \neq 0} \frac{x_{i}^{t} \Lambda_{i}^{-\frac{1}{2}} U_{i}^{t} s_{i} s_{i}^{t} U_{i} \Lambda_{i}^{-\frac{1}{2}} x_{i}}{x_{i}^{t} x_{i}}, \\
& \text { where } x_{i}=\Lambda_{i}^{\frac{1}{2}} U_{i}^{t} c_{i} .
\end{aligned}
$$


Thus the arg max is given by $x_{i}=\Lambda_{i}^{-\frac{1}{2}} U_{i}^{t} s_{i}$ and the optimal receiver structure is

$$
c_{i}=Z_{i}^{-1} s_{i} \text {. }
$$

Hence, under the MMSE receiver

$$
\begin{aligned}
\mathrm{SIR}_{i} & =s_{i}^{t} Z_{i}^{-1} s_{i} p_{i} \\
& =s_{i}^{t}\left(Z-p_{i} s_{i} s_{i}^{t}\right)^{-1} s_{i} p_{i} \\
& =s_{i}^{t}\left(Z^{-1}+\frac{Z^{-1} s_{i} s_{i}^{t} Z^{-1} p_{i}}{1-s_{i}^{t} Z^{-1} s_{i} p_{i}}\right) s_{i} p_{i} \\
& =\frac{s_{i}^{t} Z^{-1} s_{i} p_{i}}{1-s_{i}^{t} Z^{-1} s_{i} p_{i}}
\end{aligned}
$$

where we used the following formula in the second step:

$$
\left(A-x y^{t}\right)^{-1}=A^{-1}+\frac{A^{-1} x y^{t} A^{-1}}{1-y^{t} A^{-1} x}
$$

whenever the terms exist. Here $s_{i}^{t} Z^{-1} s_{i} p_{i}$ is strictly less than 1 and thus all the terms are well defined.

\section{Characterization OF USER CAPACITY}

We begin with an elementary calculation of the error of estimation using the MMSE linear receiver in terms of the signature sequences and powers of the users and derive a "conservation law" for the estimation errors.

\section{A. Conservation Law for the MMSE Receiver}

Recall the channel model in matrix form

$$
Y=S X+W
$$

where $S$ is the matrix the columns of which are the signature sequences of the users and $X$ is the vector of transmitted symbols from the users. If $\hat{X}$ is the vector MMSE estimate of $X$, a direct application of the orthogonality principle yields

$$
\hat{X}=D S^{t}\left[S D S^{t}+\sigma^{2} I\right]^{-1} Y
$$

and the covariance matrix of the error $\epsilon \equiv \hat{X}-X$ is given by

$$
K_{\epsilon}=D-D S^{t}\left[S D S^{t}+\sigma^{2} I\right]^{-1} S D
$$

where $D \equiv \operatorname{diag}\left(p_{1}, \cdots, p_{K}\right)$ is the covariance matrix of $X$. It follows that

$$
\begin{aligned}
\operatorname{trace} & \left(D^{-\frac{1}{2}} K_{\epsilon} D^{-\frac{1}{2}}\right) \\
& =K-\operatorname{trace}\left(D^{\frac{1}{2}} S^{t}\left[S D S^{t}+\sigma^{2} I\right]^{-1} S D^{\frac{1}{2}}\right) \\
& =K-\operatorname{trace}\left(S D S^{t}\left[S D S^{t}+\sigma^{2} I\right]^{-1}\right) \\
& =K-\sum_{i=1}^{N} \frac{\lambda_{i}}{\lambda_{i}+\sigma^{2}}
\end{aligned}
$$

where $\lambda_{i}$ 's are the eigenvalues of the matrix $S D S^{t}$. If we let

$$
\mathrm{MMSE}_{i} \equiv \frac{E\left[\left(\hat{X}_{i}-X_{i}\right)^{2}\right]}{p_{i}}
$$

be the (normalized) minimum mean-square error for user $i$, then (8) becomes

$$
\begin{aligned}
\sum_{i=1}^{K} \operatorname{MMSE}_{i} & =K-\sum_{i=1}^{N} \frac{\lambda_{i}}{\lambda_{i}+\sigma^{2}} \\
& >K-\operatorname{rank}(S) .
\end{aligned}
$$

There is a simple one-to-one relationship between the normalized minimum mean-square error $\mathrm{MMSE}_{i}$ of user $i$ and the SIR achieved by that user, namely, $\operatorname{SIR}_{i}$ (see (2)). Let $\hat{X}_{i}$ be the linear estimate of $X_{i}$ from $Y$ using the linear receiver $c_{i}$, i.e., $\hat{X}_{i}=c_{i}^{t} Y$. We assume that $c_{i}$ is appropriately normalized so that the error in the estimate $\operatorname{MMSE}_{i}(\alpha)=E\left[\left(\alpha \hat{X}_{i}-X_{i}\right)^{2}\right]$ is minimized at $\alpha=1$ (the corresponding (minimum) error in the estimate denoted by $\mathrm{MMSE}_{i}$ ). Then, it can be verified that

$$
\operatorname{MMSE}_{i}=\frac{1}{1+\operatorname{SIR}_{i}} .
$$

In particular, this relation holds when the receiver is the linear MMSE one, that is, $c_{i}$ is chosen according to (3). Using (9) and (11), we have

$$
\begin{aligned}
\sum_{i=1}^{K} \frac{\operatorname{SIR}_{i}}{1+\operatorname{SIR}_{i}} & =K-\sum_{i=1}^{K} \operatorname{MMSE}_{i} \\
& =\sum_{i=1}^{N} \frac{\lambda_{i}}{\lambda_{i}+\sigma^{2}} \\
& <N .
\end{aligned}
$$

The upper bound in (13) provides an upper bound to the performance of the users regardless of the signature sequences used and powers allocated-we view this as a conservation law. We can now derive the first main result of this paper: the identification of the user capacity of a single-cell S-CDMA system equipped with linear MMSE receiver. We assume that each user has the same SIR requirement $\beta$. Observe that if the number of users is less than or equal to the processing gain, the trivial choice of orthogonal signature sequences for the users ensures arbitrary SIR requirements to be met if we can scale up the power of the users. Hence, without loss of generality, we henceforth assume that the number of users is greater than the processing gain.

\section{B. User Capacity Characterization}

The following is a complete characterization of the admissibility of the users with equal SIR requirements and equipped with MMSE receivers:

Theorem 3.1: $K$ users are admissible in the system with processing gain $N$ if and only if

$$
K<N\left(1+\frac{1}{\beta}\right) \text {. }
$$

Proof: Suppose $K$ users are admissible in the system with processing gain $N$. Then, by definition, there exist sequences $s_{1}, \cdots, s_{K} \in \mathbb{S}_{1}^{N}$, positive powers $p_{1}, \cdots, p_{K}$ such that for every user $i$, we have $\operatorname{SIR}_{i} \geq \beta$, where the receiver structure is as in (3). Appealing now to (13), we have the upper bound

$$
K<N\left(1+\frac{1}{\beta}\right) .
$$

To see that this sufficient also, we provide an explicit valid allocation scheme:

Suppose $K<N\left(1+\frac{1}{\beta}\right)$. Choose the powers to be

$$
p_{i}=p=\frac{\sigma^{2}}{1+\frac{1}{\beta}-\frac{K}{N}}, \quad \forall i=1 \cdots K
$$


and sequences such that $S S^{t}=\frac{K}{N} I$. Then, using (5), $\forall i=$ $1 \cdots K$

$$
\begin{aligned}
\frac{\mathrm{SIR}_{i}}{1+\mathrm{SIR}_{i}} & =s_{i}^{t}\left(p S S^{t}+\sigma^{2} I\right)^{-1} s_{i} p \\
& =s_{i}^{t}\left(\frac{K}{N} p I+\sigma^{2} I\right)^{-1} s_{i} p \\
& =\frac{N p}{K p+N \sigma^{2}} \\
& =\frac{\beta}{1+\beta} .
\end{aligned}
$$

Hence for each user $i$, we have $\operatorname{SIR}_{i}=\beta$ and the $K$ users are admissible in the system with processing gain $N$. We need to show the existence of sequences such that $S S^{t}=\frac{K}{N} I$. We begin with some definitions.

Definition 3.1: For any $x=\left(x_{1}, \cdots, x_{n}\right) \in \mathbb{R}^{n}$, let

$$
x_{[1]} \geq \cdots \geq x_{[n]}
$$

denote the components of $x$ in decreasing order, called the order statistics of $x$.

Majorization makes precise the vague notion that the components of a vector $x$ are "less spread out" or "more nearly equal" than are the components of a vector $y$ by the statement $x$ is majorized by $y$.

Definition 3.2: For $x, y \in \mathbb{R}^{n}$, say that $x$ is majorized by $y$ (or $y$ majorizes $x$ ) if

$$
\begin{aligned}
\sum_{i=1}^{k} x_{[i]} & \leq \sum_{i=1}^{k} y_{[i]}, \quad k=1 \cdots n-1 \\
\sum_{i=1}^{n} x_{[i]} & =\sum_{i=1}^{n} y_{[i]},
\end{aligned}
$$

A comprehensive reference on majorization and its applications is [11]. A simple (trivial, but important) example of majorization between two vectors is the following:

Example 3.1: For every $a \in \mathbb{R}^{n}$ such that $\sum_{i=1}^{n} a_{i}=1$

$$
\left(a_{1}, \cdots, a_{n}\right) \text { majorizes }\left(\frac{1}{n}, \frac{1}{n}, \cdots, \frac{1}{n}\right) \text {. }
$$

It is well known that the sum of diagonal elements of a matrix is equal to the sum of its eigenvalues. When the matrix is symmetric the precise relationship between the diagonal elements and the eigenvalues is that of majorization.

Lemma 3.1 ([11, Theorems 9.B.1 and 9.B.2]): Let $H$ be a symmetric matrix with diagonal elements $h_{1}, \cdots, h_{n}$ and eigenvalues $\lambda_{1}, \cdots, \lambda_{n}$ we have

$$
\left(\lambda_{1}, \cdots, \lambda_{n}\right) \text { majorizes }\left(h_{1}, \cdots, h_{n}\right) .
$$

That $h=\left(h_{1}, \cdots, h_{n}\right)$ and $\lambda=\left(\lambda_{1}, \cdots, \lambda_{n}\right)$ cannot be compared by an ordering stronger than majorization is the consequence of the following converse: If $h_{1} \geq \cdots \geq h_{n}$ and $\lambda_{1} \geq \cdots \lambda_{n}$ are $2 n$ numbers such that $\lambda$ majorizes $h$, then there exists a real symmetric matrix $H$ with diagonal elements $h_{1}, \cdots, h_{n}$ and eigenvalues $\lambda_{1}, \cdots, \lambda_{n}$.
We use these notions and results to construct sequences such that $S S^{t}=\frac{K}{N} I$. The vector $(1, \cdots, 1)$ in $\mathbb{R}^{K}$ is majorized by the vector with $N$ entries equal to $\frac{K}{N}$ and the remaining $K-N$ entries equal to 0 (this is a simple application of Example 3.1). Now, appealing to Lemma 3.1, there exists a symmetric matrix, say $P$, with unit diagonal entries and eigenvalues $\frac{K}{N}$ and 0 with multiplicities (both algebraic and geometric) equal to $N$ and $K-N$, respectively. Let $v_{1}, \cdots, v_{N} \in$ $\mathbb{R}^{1 \times K}$ be orthonormal eigenvectors of $P$ corresponding to the eigenvalue $\frac{K}{N}$. Denoting the matrix $S=\left[s_{1}, \cdots, s_{K}\right]$, we allocate sequences for the users as follows:

$$
S=\sqrt{\frac{K}{N}}\left[\begin{array}{c}
v_{1} \\
v_{2} \\
\vdots \\
v_{N}
\end{array}\right] .
$$

Then, note that $S S^{t}=\frac{K}{N} I$. These sequences were first identified in [12] (but in their context the sequences were in $\{1,-1\}^{N}$ ) and the authors referred to such $s_{1}, \cdots, s_{K}$ as WBE sequences, sequences that meet the so-called Welchbound equality (see [25]). We shall henceforth assume that the WBE sequences are in $\mathbb{S}_{1}^{N-1}$.

\section{Observations on Valid Allocations}

Below, we observe some properties of valid allocations in general and in particular the specific valid allocation we demonstrated in the proof of Theorem 3.1.

1) Suppose $K$ users are admissible in the system with processing gain $N$. Let $s_{1}, \cdots, s_{K}$ be a valid allocation of sequences. Then, with these sequences fixed, among all valid allocations of powers (i.e., with this power allocation $\operatorname{SIR}_{i} \geq \beta_{i}$ ) there exists a component-wise minimal power allocation (see [18]) and with this power allocation, $\operatorname{SIR}_{i}=\beta_{i}$. Hence, when the sequences are WBE sequences, it follows that the power allocation

$$
p_{i}=p=\frac{\sigma^{2}}{1+\frac{1}{\beta}-\frac{K}{N}}
$$

is the component-wise minimal power solution.

2) We shall now focus on this specific allocation scheme of WBE sequences and corresponding component-wise minimal powers. This scheme allocates the smallest sum of powers among all valid allocations. We shall show this property in Section IV when we revisit user capacity with power constraints.

3) With this allocation, the MMSE receiver for user $i$ is given by, following (3),

$$
\begin{aligned}
c_{i} & =Z_{i}^{-1} s_{i} \\
& =\left(\sigma^{2} I+\sum_{j \neq i} p_{j} s_{j} s_{j}^{t}\right)^{-1} s_{i} \\
& =\left(\sigma^{2} I-p s_{i} s_{i}^{t}+p S S^{t}\right)^{-1} s_{i} \\
& =\left(\sigma^{2} I-p s_{i} s_{i}^{t}+\frac{p K}{N} I\right)^{-1} s_{i} \\
& =a s_{i}
\end{aligned}
$$


where $a$ is a constant (which can be shown to be equal to $\left.\frac{\beta}{p}\right)$. Thus the optimal linear filter in this situation is just a scaled version of the matched filter. This observation allows us to conclude that the user capacity of a system equipped with the a priori inferior matched filter receiver is the same as that of the linear MMSE receiver:

Corollary 3.2: $K$ users are admissible in the system with processing gain $N$ and equipped with matched filter receivers if and only if

$$
K<N\left(1+\frac{1}{\beta}\right)
$$

\section{Comparison with Random Sequences}

It is interesting to compare this result with the corresponding characterization of user capacity of a system with random signature sequences carried out in [17]. The results in [17] are asymptotic and are valid for a large system (i.e., a system with a large processing gain and large number of users). We focus on the system where each user has SIR requirement $\beta$, the number of users is $K$, and the processing gain is $N$ and consider the regime $K \rightarrow \infty, N \rightarrow \infty$, and $\frac{K}{N} \rightarrow \alpha$. Appealing to the results in [17] we conclude as follows:

1) Suppose MMSE linear receivers are used. Then, ([17, Sec. 5]) the SIR of each user converges (in probability) to a constant and (with appropriate power control) this constant is at least the target requirement $\beta$ if and only if $\alpha<1+\frac{1}{\beta}$. Thus for a large system, this suggests that using random sequences is as good as using the optimal WBE sequences (in the context of user capacity) for the signature sequences of the users.

From (10), note that the total normalized MMSE errors of the users is a constant, independent of the relative powers of the users and depends very weakly on the signature sequences. To minimize the maximum MMSE among all users (or equivalently, to maximize the minimum SIR's), it is, therefore, optimal to have symmetry among the users such that they have the same MMSE. This was achieved using equal received power and the WBE sequences described earlier. However, this "symmetrization" can also be achieved asymptotically when random sequences are used, since it is shown in [17] that (with appropriate power control) the SIR's of all users will converge to the same number. But maximizing the minimum SIR's is equivalent to maximizing the number of users in a system with given equal SIR requirements. Hence, random sequences and the WBE sequences yield the same user capacity asymptotically.

2) For the scenario of matched filters, following [17, Sec. 5], the SIR of each user converges (in probability) to a constant and this constant is at least the requirement $\beta$ if and only if $\alpha<\frac{1}{\beta}$. Thus one user per degree of freedom is lost asymptotically when random sequences are used. A conservation law similar to the one for MMSE receivers (as in (12)) is lacking for the matched filter receivers.

\section{POWER CONSTRAINT AND USER CAPACITY}

Our model has not included any constraints on the allocated received power of the users; we now include a received power constraint in our model. In this section we precisely quantify the loss in the user capacity due to the received power constraint; this loss will be a function of both the constraint and the power of the background Gaussian noise. One way to justify the constraint on the received power of the users is by considering average transmit power constraint of the users. If one is able to adopt a model of fading for the users that is ergodic (with the same mean fading) and independent, an average transmit power constraint on the users translates into a received power constraint.

\section{A. User Capacity with Power Constraints}

We define admissibility of $K$ users (each having SIR requirement $\beta$ ) in the system with processing gain $N$ and power constraint $\bar{P}$ as being able to allot $i$ signature sequence $s_{i}$ and power $p_{i} \leq \bar{P}$ for every user such that the achieved $\mathrm{SIR}_{i}$ (given in (4)) is at least the target value $\beta$. If the system has just one user, then the minimum power required by that single user to meet its SIR requirement is $\beta \sigma^{2}$. To prevent a degenerate situation, we shall assume that $\bar{P} \geq \beta \sigma^{2}$. As before, we assume that the number of users is greater than the processing gain. Our main result in this section is to precisely identify the loss in user capacity by including such a power constraint:

Theorem 4.1: $K$ users (each having SIR requirement $\beta$ ) are admissible in the system with processing gain $N$ and average received power constraint $\bar{P}$ if and only if

$$
K<N\left(1+\frac{1}{\beta}-\frac{\sigma^{2}}{\bar{P}}\right)
$$

Proof: We first show the necessity. Suppose $K$ users are admissible. Then there exist signature sequences $s_{1}, \cdots, s_{K} \in$ $\mathbb{S}_{1}^{N}$, positive powers $p_{1}, \cdots, p_{K} \leq \bar{P}$, such that for every user $i$, we have $\operatorname{SIR}_{i} \geq \beta$. From (12), we have the conservation law

$$
\sum_{i=1}^{K} \frac{\operatorname{SIR}_{i}}{1+\operatorname{SIR}_{i}}=\sum_{i=1}^{N} \frac{\lambda_{i}}{\lambda_{i}+\sigma^{2}}
$$

where $\lambda_{1}, \cdots, \lambda_{N}$ are the eigenvalues of the matrix $S D S^{t}$. We note that

$$
\begin{aligned}
\operatorname{tr}(\Lambda) & =\operatorname{tr}\left(S D S^{t}\right) \\
& =\operatorname{tr}(D)
\end{aligned}
$$

where the second equality follows by some algebra and noting that the columns of $S$ have unit $l_{2}$ norm. Now, if we let $p^{*}=\frac{1}{N} \sum_{i=1}^{K} p_{i}$, then the vector $\left(p^{*}, p^{*}, \cdots, p^{*}\right)$ is majorized by the vector $\left(\lambda_{1}, \cdots, \lambda_{N}\right)$ (see Example 3.1). We need the following definition.

Definition 4.1: A real-valued function $\phi: \mathbb{R}^{n} \rightarrow \mathbb{R}$ is said to be Schur-concave if for all $x, y \in \mathcal{R}^{n}$ such that $y$ majorizes $x$ we have $\phi(x) \geq \phi(y)$. We say that $\phi$ is Schur-convex if $-\phi$ is Schur-concave.

An important class of Schur-convex functions is the following ([11, Theorem 3.C.1]). 
Example 4.1: If $g: \mathbb{R} \rightarrow \mathbb{R}$ is convex then the symmetric convex function

$$
\phi(x)=\sum_{i=1}^{n} g\left(x_{i}\right)
$$

is Schur-convex. By definition, if $g: \mathbb{R} \rightarrow \mathbb{R}$ is concave then the symmetric concave function

$$
\phi(x)=\sum_{i=1}^{n} g\left(x_{i}\right)
$$

is Schur-concave.

Observe that the map $x \mapsto \frac{x}{x+\sigma^{2}}$ is concave in $x$ and hence the symmetric concave map

$$
\left(\lambda_{1}, \cdots, \lambda_{N}\right) \mapsto \sum_{i=1}^{N} \frac{\lambda_{i}}{\lambda_{i}+\sigma^{2}}
$$

is Schur-concave (see Example 4.1). Then we have

$$
\begin{aligned}
\sum_{i=1}^{N} \frac{\lambda_{i}}{\lambda_{i}+\sigma^{2}} & \leq \sum_{i=1}^{N} \frac{p^{*}}{p^{*}+\sigma^{2}} \\
& =\frac{N p^{*}}{p^{*}+\sigma^{2}} .
\end{aligned}
$$

By hypothesis, $\operatorname{SIR}_{i} \geq \beta$ and hence

$$
K \frac{\beta}{1+\beta} \leq \frac{N p^{*}}{p^{*}+\sigma^{2}}
$$

which implies that

$$
p^{*} \geq \frac{K \beta \sigma^{2}}{N(1+\beta)-K \beta} .
$$

Since each $p_{i} \leq \bar{P}$ it follows that $p^{*} \leq \frac{K}{N \bar{P}}$. We then conclude that $K<N\left(1+\frac{1}{\beta}-\frac{\sigma^{2}}{\bar{P}}\right)$.

To see this is sufficient as well, suppose $K<N\left(1+\frac{1}{\beta}-\frac{\sigma^{2}}{\bar{P}}\right)$. We shall use the same allocation scheme as in the proof of Theorem 3.1, namely, signature sequences to be WBE sequences and powers

$$
p_{i}=p=\frac{\sigma^{2}}{1+\frac{1}{\beta}-\frac{K}{N}} .
$$

We have, as in the proof of Theorem 3.1, the achieved SIR of each user is equal to $\beta$. We only need to verify that our choice of powers does not exceed the constraint $\bar{P}$. Using the prior $K<N\left(1+\frac{1}{\beta}-\frac{\sigma^{2}}{\bar{P}}\right)$ we have for each user power

$$
p_{i}=\frac{\sigma^{2}}{1+\frac{1}{\beta}-\frac{K}{N}} \leq \bar{P} .
$$

Thus the $K$ users are admissible in the system.

The proof of the characterization of user capacity allows us to conclude an optimality property of the specific allocation scheme used above: suppose $K$ users are admissible in the system with processing gain $N$. Then for any valid allocation (signature sequences $S$ and powers $p_{1}^{S}, \cdots, p_{K}^{S}$ ), from (18), we have that

$$
\sum_{i=1}^{K} p_{i}^{S} \geq \frac{K \sigma^{2}}{1+\frac{1}{\beta}-\frac{K}{N}}
$$

For the allocation in the proofs of Theorems 3.1 and 4.1, the lower bound in (19) is met with equality. We draw the following conclusions.

1) The allocation of WBE sequences and powers all equal to

$$
p=\frac{\sigma^{2}}{1+\frac{1}{\beta}-\frac{K}{N}}
$$

gives the smallest sum of received powers among all valid allocations. In this sense, this allocation is optimal.

2) Since with this allocation the MMSE linear receiver for each user is just the corresponding scaled matched filter receiver (as seen in (15)), we have the following corollary.

Corollary 4.2: $K$ users (each having SIR requirement $\beta$ ) are admissible in the system with processing gain $N$ and received power constraint $\bar{P}$ and equipped with matched filter receivers if and only if

$$
K<N\left(1+\frac{1}{\beta}-\frac{\sigma^{2}}{\bar{P}}\right) .
$$

\section{B. Comparison with Random Sequences}

We saw from the comparison in Section III that asymptotically (in a large system, where number of users $K \rightarrow \infty$, processing gain $N \rightarrow \infty$, and $\frac{K}{N} \rightarrow \alpha$ ) there is no loss in user capacity with MMSE receivers while using random sequences. It is interesting to compare the results when there is a received power constraint. From the results in [17], we summarize.

1) From [17, Sec. 5] we conclude that with a received power constraint $\bar{P}$, users have their SIR requirement $\beta$ achieved (using MMSE receiver) if and only if

$$
\alpha<1+\frac{1}{\beta}-(1+\beta) \frac{\sigma^{2}}{\bar{P}} .
$$

The user capacity is thus strictly less than that with optimal sequences, when the power constraint is finite. To understand why, we can appeal to (9)

$$
\sum_{i=1}^{K} \operatorname{MMSE}_{i}=K-\sum_{i=1}^{N} \frac{\lambda_{i}}{\lambda_{i}+\sigma^{2}}
$$

where $\lambda_{i}$ 's are the eigenvalues of the matrix $S D S^{t}$. When the background noise power is small compared to the received powers, any set of sequences which symmetrizes the MMSE for all users are optimal. When $\sigma^{2}$ is nonnegligible, good sequences should also minimize the right-hand side as well. The WBE sequences achieve that by making the eigenvalues least "spread out," i.e., all the same. Using random sequences, the eigenvalues are more spread out, resulting in a user capacity penalty when $\sigma^{2}$ is nonnegligible.

2) When matched filters are used, following [17, Sec. 5], we conclude that users can meet their target SIR requirement $\beta$ if and only if $\alpha<\frac{1}{\beta}-\frac{\sigma^{2}}{\bar{P}}$ when the received power constraint is $\bar{P}$. Thus there is a loss in 
user capacity strictly more than one user per degree of freedom when using random sequences when compared with the user capacity achieved with optimal sequences.

\section{Multiple Classes, User \\ CAPACITY, AND OPTIMAL ALLOCATIONS}

In this section we consider the situation when the users have different SIR requirements and completely generalize the results of the previous two sections. This level of generality of allowing users to have different SIR requirements caters to data and voice users, sharing the common system and having different SIR requirements. As earlier, we shall assume, without loss of generality, that the number of users is bigger than the processing gain.

\section{A. User Capacity Characterization}

We first define admissibility of $K$ users (with SIR requirements $\left.\beta_{1}, \beta_{2}, \cdots, \beta_{K}\right)$ in the system with processing gain $N$ as being able to allot signature sequences and powers for the users such that for each user $i$ the SIR achieved by the MMSE receiver for that user (as in (4)) is greater than or equal to $\beta_{i}$ (such allocations are called valid). Our main result of this section is an analog of Theorem 3.1; a complete characterization of admissibilit:.

Theorem 5.1: $K$ users (with SIR requirements $\beta_{1}, \beta_{2}, \cdots$, $\left.\beta_{K}\right)$ are admissible in the system with processing gain $N$ if and only if

$$
\sum_{i=1}^{K} \frac{\beta_{i}}{1+\beta_{i}}<N
$$

Proof: This result motivates the consideration of the quantity $\frac{\beta}{1+\beta}$ as the effective bandwidth of a user with SIR requirement $\beta$. Then the criterion of admissibility has a simple interpretation: Users are admissible if and only if the sum of their effective bandwidths is less than the processing gain of the system. Let us denote the effective bandwidth by $e(\beta)=\frac{\beta}{1+\beta}$.

We first show the necessity: since $K$ users are admissible, by definition, there exists a valid allocation of signature sequences $s_{i}$ and powers $p_{i}$. Since $\operatorname{SIR}_{i} \geq \beta_{i}$, and the effective bandwidth is a monotonic function of the SIR requirement, from (12) we have

$$
\sum_{i=1}^{K} \frac{\beta_{i}}{1+\beta_{i}}<N
$$

We show that this is also sufficient by explicitly demonstrating a valid allocation. Restricted to the context of this proof, we assume for notational simplicity that the SIR requirements of the users are ordered, i.e., $\beta_{1} \geq \beta_{2} \geq \cdots \geq \beta_{K}$.

We look for the unique $k \in\{0, \cdots, N-1\}$ such that

$$
(N-k) e\left(\beta_{k}\right)>\sum_{j=k+1}^{K} e\left(\beta_{j}\right) \geq(N-k) e\left(\beta_{k+1}\right) \text {. }
$$

Say that the users $1, \cdots, k$ are oversized. If $k=0$, then no user is oversized (and this happens when $N e\left(\beta_{1}\right) \leq \sum_{j=1}^{K} e\left(\beta_{j}\right)$ ).
Observe that oversized users have effective bandwidth large relative to the effective bandwidths of the other users. A simple recursive scheme to enumerate the oversized users follows directly from the definition in (21).

Let $e_{i} \in \mathbb{R}^{N}$ be the vector $(0,0, \cdots, 0,1,0, \cdots, 0)$ with the entry 1 being in the $i$ th position. Then $e_{1}, \cdots, e_{N}$ form an orthonormal basis for $\mathbb{R}^{N}$. Recall that users $1, \cdots, k$ are oversized, and we know that $k<N$. Consider the following allocation of powers and sequences:

1) For oversized users, we allocate independent channels, i.e., for users in $1, \cdots, k$, we allocate the signature sequences $e_{1}, \cdots, e_{k}$ and powers $p_{i}=\sigma^{2} \beta_{i}$.

2) For nonoversized users we allocate sequences from the subspace $\operatorname{span}\left\{e_{k+1}, \cdots, e_{K}\right\}$ which has dimension $N-k$. From (21), it follows that

$$
\sum_{i=k+1}^{K} e\left(\beta_{i}\right) \geq(N-k) e\left(\beta_{k+1}\right)
$$

and hence the vector with with $K-N$ entries equal to 0 and the other $N-k$ entries equal to

$$
\frac{1}{N-k} \sum_{j=k+1}^{K} e\left(\beta_{j}\right)
$$

majorizes the vector $\left(e\left(\beta_{j}\right), j=k+1, \cdots, K\right)$ (see Example 3.1). Now, appealing to Lemma 3.1, there exists a symmetric matrix, say $P$, with diagonal entries

$$
\left(e\left(\beta_{j} t\right), \forall j=k+1, \cdots, K\right)
$$

and eigenvalues

$$
\frac{1}{N-k} \sum_{j=k+1}^{K} e\left(\beta_{j}\right)
$$

and 0 with multiplicities (both algebraic and geometric) equal to $N-k$ and $K-N$, respectively. Let $v_{1}, \cdots, v_{N-k} \in \mathbb{R}^{1 \times K-k}$ be orthonormal eigenvectors of $P$ corresponding to the eigenvalue

$$
\frac{1}{N-k} \sum_{j=k+1}^{K} e\left(\beta_{j}\right)
$$

Now, for every user $j=k+1, \cdots, K$ choose power proportional to its effective bandwidth, namely,

$p_{j}=c e\left(\beta_{j}\right), \quad$ where constant $c=\frac{(N-k) \sigma^{2}}{N-k-\sum_{l=k+1}^{K} e\left(\beta_{l}\right)}$

Observe that $c$ is positive by the hypothesis

$$
\sum_{i=1}^{K} e\left(\beta_{i}\right)=\sum_{i=1}^{K} \frac{\beta_{i}}{1+\beta_{i}}<N
$$

Let $\tilde{D}$ be the diagonal matrix with entries $p_{j}, j=k+$ $1, \cdots, K$. For $j=k+1, \cdots, K$, define sequences $\tilde{s}_{j} \in$ 
$\mathbb{R}^{1 \times(K-k)}$ and the matrix $\widetilde{S}=\left[\tilde{s}_{j} j=k+1, \cdots, K\right]$ as

$$
\tilde{S}=\frac{\sqrt{c \sum_{j=k+1}^{K} e\left(\beta_{j}\right)}}{\sqrt{N-k}}\left[\begin{array}{c}
v_{1} \\
v_{2} \\
\vdots \\
v_{N-k}
\end{array}\right] \tilde{D}^{\frac{1}{2}} .
$$

Since the diagonal entries of $\tilde{S}^{t} \tilde{S}$ are unity, $\tilde{s}_{j}$ have unit norm for every $j=k+1, \cdots, K$. Also, note that

$$
\tilde{S} \tilde{D} \tilde{S}^{t}=\frac{c \sum_{j=k+1}^{K} e\left(\beta_{j}\right)}{N-k} I .
$$

We shall denote such sequences as generalized WBE sequences. Note that when $\tilde{D}$ is a scaled identity, the sequences reduce to the WBE sequences. The Appendix discusses the construction of generalized WBE sequences.

Now allocate signature sequences for users not oversized as follows:

$$
s_{j}=\left(0, \cdots, 0, \tilde{s}_{j}\right) \in \mathbb{S}_{1}^{N-1}, \quad \forall j=k+1, \cdots, K .
$$

With this allocation of sequences and powers we now have

$$
\begin{aligned}
Z & =S D S^{t}+\sigma^{2} I \\
& =\left[\begin{array}{cc}
\sigma^{2} \operatorname{diag}\left\{1+\beta_{i}, i=1, \cdots, k\right\} & 0 \\
0 & \sigma^{2}+\frac{c \sum_{j=k+1}^{K} e\left(\beta_{j}\right)}{N-k} I
\end{array}\right] .
\end{aligned}
$$

Substituting in (5)

$$
\begin{aligned}
\mathrm{SIR}_{i} & =\beta_{i}, \quad \forall i=1, \cdots, k \\
\operatorname{SIR}_{j} & =\frac{s_{j}^{t} Z^{-1} s_{j} p_{j}}{1-s_{j}^{t} Z^{-1} s_{j} p_{j}}, \quad \forall j=k+1, \cdots, K \\
& =\frac{(N-k) p_{j}}{(N-k) \sigma^{2}+c \sum_{l=k+1}^{K} e\left(\beta_{l}\right)-(N-k) p_{j}} \\
& =\beta_{j} .
\end{aligned}
$$

Thus each user has its SIR requirement met which completes the proof.

This characterization of admissibility along with the valid allocation scheme above allows us to make the following remarks.

1) It is interesting to observe that the linearity of the boundary of the user capacity is a consequence of (10), that the total minimum mean-square errors of the users is a constant independent of the received powers and dependent very weakly on the signature sequences. This also explains why here, as in the single class case, random sequences achieve asymptotically (as the processing gain gets large) the same performance as optimal sequences.

2) In practice, one can imagine a small number of different SIR requirements of the users (say two or three). We introduce the notion of different "classes" of users; all users of the same class have a common SIR requirement. We assume there are $L$ classes (fixed) and users of class $l$ have SIR requirement $\beta_{l}$. We know (from Section III) that $N$ degrees of freedom can support $\left\lfloor\left(1+\frac{1}{\beta}\right) N\right\rfloor$ users each with SIR requirement $\beta$. This suggests that we could "channelize" the system such that users of different classes do not interfere with each other and asymptotically achieve $\alpha_{l}$ users of class $l$ per degree of freedom whenever $\sum_{l=1}^{L} \frac{\alpha_{l}, \beta_{l}}{1+\beta_{l}}<1$. This is indeed true and the following statement can be verified: We can admit at least $k_{l}=\left\lfloor\alpha_{l} N-1-\frac{1}{\beta_{l}}\right\rfloor$ users of class $l$ in a system with processing gain $N$ where $\alpha_{1}, \cdots, \alpha_{N}$ positive such that $\sum_{l=1}^{L} \frac{\alpha_{l} \beta_{l}}{1+\beta_{l}}<1$.

3) We can identify two important situations when there are no oversized users:

a) When all the SIR requirements are identical, then no user is oversized. This is the result contained in Theorem 3.1.

b) When there are at least as many users in each class as the processing gain of the system then it is straightforward to see that that there are no oversized users. Suppose class $l$ has $K_{l} \geq N$ users and $\sum_{l=1}^{N} \frac{K_{l} \beta_{l}}{1+\beta_{l}}<N$. Then, we can make a familiar valid allocation: signature sequences for the $K_{l}$ users of class $l$ to be WBE sequences (this can be done by the hypothesis that $K_{l} \geq N$ ) and powers the same for every user $i$ of class $l$ to be

$$
p_{i}^{l}=p^{l}=\frac{\beta_{l}}{1+\beta_{l}} \frac{N \sigma^{2}}{N-\sum_{j=1}^{L} \frac{K_{j} \beta_{j}}{1+\beta_{j}}} .
$$

The SIR of user $i$ of class $l$, as in (5), can be verified to be exactly $\beta$. This ensures that for every class $l$, $K_{l}$ users of that class are admissible in the system.

4) In the proof of Theorem 5.1, the assumption that the SIR requirements of the users be ordered was for notational simplicity. Equivalently, the notion of oversized users could be introduced (with no assumption on the ordering) as below: ${ }^{1}$ User $i$ is defined to be oversized if

$$
e\left(\beta_{i}\right)>\frac{\sum_{j=1}^{K} e\left(\beta_{j}\right) 1_{\left\{e\left(\beta_{i}\right)>e\left(\beta_{j}\right)\right\}}}{N-\sum_{j=1}^{K} 1_{\left\{e\left(\beta_{j}\right) \geq e\left(\beta_{i}\right)\right\}}} .
$$

Denote the set of oversized users as $\mathcal{K}$. Then, (21) can be written in this notation as

$$
\begin{aligned}
(N-|\mathcal{K}|) \min _{i \in \mathcal{K}} e\left(\beta_{i}\right)>\sum_{j \notin \mathcal{K}} e\left(\beta_{j}\right) & \\
& \geq(N-|\mathcal{K}|) \max _{i \notin \mathcal{K}} e\left(\beta_{i}\right) .
\end{aligned}
$$

We observe that there can be at most $N-1$ oversized users and that if user $i$ is oversized then every user with SIR requirement at least $\beta_{i}$ is also oversized. In the rest of the paper we shall adhere to this notation.

5) For the specific valid allocation demonstrated in the proof of Theorem 5.1, we can calculate the MMSE

\footnotetext{
${ }^{1}$ We would like to thank Prof. Sergio Verdú for suggesting this terminology.
} 
receiver for the users (from (3)) to be

$$
\begin{aligned}
c_{i} & =s_{i} \quad \forall i \in \mathcal{K} \\
c_{j} & =Z_{j}^{-1} s_{j} \quad \forall j \notin \mathcal{K} \\
& =\left(\sigma^{2} I+\sum_{k \neq j} p_{k} s_{k} s_{k}^{t}\right)^{-1} s_{j} \\
& =\left(\sigma^{2} I-p_{j} s_{j} s_{j}^{t}+S D S^{t}\right)^{-1} s_{j} \\
& =\left(\sigma^{2} I-p_{j} \tilde{s}_{j} \tilde{s}_{j}^{t}+\tilde{S} \tilde{D}_{\hat{S}}^{t}\right)^{-1} \tilde{s}_{j} \text { from (24) } \\
& =\left(\sigma^{2} I-p_{j} \tilde{s}_{j} \tilde{s}_{j}^{t}+\frac{c \sum_{l \notin \mathcal{K}} e\left(\beta_{l}\right)}{N} I\right)^{-1} s_{j} \text { from }(23) \\
& =a_{j} s_{j}
\end{aligned}
$$

where $a_{j}$ is a constant (which is easily seen to be $\left.\left(1+\beta_{j}\right)\left(1-\frac{1}{N-|\mathcal{K}|} \sum_{k \notin \mathcal{K}} e\left(\beta_{k}\right)\right)\right)$. Thus the MMSE receiver is just the scaled matched filter receiver for each user. This allows us to conclude, exactly as in Section III, that there is no loss in user capacity when we restrict the system to use the a priori inferior matched filter. To emphasize

Corollary 5.2: $K$ users (with SIR requirements $\left.\beta_{1}, \cdots, \beta_{K}\right)$ are admissible in the system with processing gain $N$ and equipped with matched filter receivers if and only if

$$
\sum_{i=1}^{K} \frac{\beta_{i}}{1+\beta_{i}}<N
$$

\section{B. Optimal Allocation of Powers and Sequences}

We proved Theorem 5.1 by explicitly demonstrating a valid allocation scheme. We now identify the nature of optimality of this allocation scheme. This is a generalization of the ideas in Section IV to the situation when users have different SIR requirements.

The key observation used in Section IV to characterize the optimality of WBE sequences was (9) which related the eigenvalues of $S D S^{t}$ and the MMSE's of the users (and hence the attained SIR's of the users; using (11)). We now strengthen (9) to obtain the precise relationship between the eigenvalues of $S D S^{t}$ and the attained SIR's of the users using the MMSE receiver structure.

Fix $K>N$, the signature sequence matrix $S$, and the diagonal matrix of user powers $D$. Suppose the MMSE receiver structure is used and the attained SIR's of the users are $\beta_{1}, \cdots, \beta_{K}$. Then appealing to (6) and (11) we have that the diagonal entries of the matrix $D^{\frac{1}{2}} S^{t}\left[S D S^{t}+\sigma^{2} I\right]^{-1} S D^{\frac{1}{2}}$ are

$$
\frac{\beta_{1}}{1+\beta_{1}}, \cdots, \frac{\beta_{K}}{1+\beta_{K}}
$$

Now, if we denote the eigenvalues of $S D S^{t}$ by $\lambda_{1}, \cdots, \lambda_{N}$ it is straightforward to verify that the $K \times K$ matrix $D^{\frac{1}{2}} S^{t}\left[S D S^{t}+\sigma^{2} I\right]^{-1} S D^{\frac{1}{2}}$ has $N$ eigenvalues equal to

$$
\frac{\lambda_{1}}{\sigma^{2}+\lambda_{1}}, \cdots, \frac{\lambda_{N}}{\sigma^{2}+\lambda_{N}}
$$

and the remaining $K-N$ eigenvalues equal to 0 . Hence, referring to Lemma 3.1

$$
\begin{aligned}
& \left(\frac{\lambda_{1}}{\sigma^{2}+\lambda_{1}}, \cdots, \frac{\lambda_{N}}{\sigma^{2}+\lambda_{N}}, 0 \cdots, 0\right) \\
& \quad \text { majorizes }\left(\frac{\beta_{1}}{1+\beta_{1}}, \cdots, \frac{\beta_{K}}{1+\beta_{K}}\right) .
\end{aligned}
$$

Observe that the conservation law mentioned in (9) follows directly from (27) above. The statement that (27) is the precise relationship between the eigenvalues of $S D S^{t}$ and the attained SIR's of the users is made clear by the following observation.

Suppose we are given $y_{1}, \cdots, y_{N} \in[0,1)$ and $\beta_{1}, \cdots, \beta_{K} \geq$ 0 such that

$$
\left(y_{1}, \cdots, y_{N}, 0, \cdots 0\right) \text { majorizes }\left(\frac{\beta_{1}}{1+\beta_{1}}, \cdots, \frac{\beta_{K}}{1+\beta_{K}}\right) \text {. }
$$

Then the claim is that there exists an allocation of signature sequences $S$ and user powers $D$ such that

$$
S D S^{t} \text { has eigenvalues } \frac{\sigma^{2} y_{1}}{1-y_{1}}, \cdots, \frac{\sigma^{2} y_{N}}{1-y_{N}} \text {. }
$$

The attained SIR's of the users are $\beta_{1}, \cdots, \beta_{K}$.

To see this, consider the following construction of $S$ and $D$. Given $y_{1}, \cdots, y_{N} \in[0,1)$ and $\beta_{1}, \cdots, \beta_{K} \geq 0$ satisfying (28), by an appeal to Lemma 3.1, there exists a $K \times K$ symmetric matrix $H$ with diagonal entries

$$
\frac{\beta_{1}}{1+\beta_{1}}, \cdots, \frac{\beta_{K}}{1+\beta_{K}}
$$

and eigenvalues $y_{1}, \cdots, y_{N}, 0, \cdots, 0$. Denote $\lambda_{i}=\frac{\sigma^{2} y_{i}}{1-y_{i}}$ for each $i=1 \cdots N$ and let $\Lambda=\operatorname{diag}\left\{\lambda_{1}, \cdots, \lambda_{N}\right\}$. Let $\hat{U}$ be the $K \times N$ matrix with columns the normalized eigenvectors of $H$ corresponding to the eigenvalues $y_{1}, \cdots, y_{N}$. Then

$$
H=\hat{U}\left(\Lambda+\sigma^{2} I\right)^{-1} \Lambda \hat{U}^{t} \text {. }
$$

Define $p_{1}, \cdots, p_{K}$ to be the diagonal entries of the $K \times K$ matrix $\hat{U} \Lambda \hat{U}^{t}$ and $D$ to be the diagonal matrix with diagonal entries $p_{1}, \cdots, p_{K}$. Now define the signature sequence matrix $S$ by

$$
S=Q \Lambda^{\frac{1}{2}} \hat{U}^{t} D^{-\frac{1}{2}}
$$

for some orthonormal $N \times N$ matrix $Q$. Then observe the following.

1) The columns of $S$ have unit norm.

2) $S D S^{t}=Q \Lambda^{\frac{1}{2}} \hat{U}^{t} U \Lambda^{\frac{1}{2}} Q^{t}=Q \Lambda Q^{t}$ has eigenvalues $\lambda_{1}, \cdots, \lambda_{N}$ be definition.

3) The SIR's attained by the users are $\beta_{1}, \cdots, \beta_{K}$. To see this, note that

$$
\begin{aligned}
D^{\frac{1}{2}} S^{t}\left[S D S^{t}+\sigma^{2} I\right]^{-1} S D^{\frac{1}{2}} & =\hat{U} \Lambda^{\frac{1}{2}}\left(\Lambda+\sigma^{2} I\right)^{-1} \Lambda^{\frac{1}{2}} \hat{U}^{t} \\
& =H \quad \text { using }(31)
\end{aligned}
$$

and by definition the diagonal entries of $H$ are

$$
\frac{\beta_{1}}{1+\beta_{1}}, \cdots, \frac{\beta_{K}}{1+\beta_{K}} \text {. }
$$


Hence by construction we have demonstrated the existence of signature sequences $S$ and powers $D$ so that both (29) and (30) are met.

We shall now use the observation in (27) and (28) to characterize the optimality of our signature sequence and power allocation. Now suppose we are given that the necessary condition $\sum_{i=1}^{K} \frac{\beta_{i}}{1+\beta_{i}}<N$ in Theorem 5.1 is true. Then by Theorem 5.1, there exist some $S$ and some $D$ such that the achieved SIR's of the users are $\beta_{1}, \cdots, \beta_{K}$. If we denote $\lambda_{1}, \cdots, \lambda_{N}$ as the eigenvalues of $S D S^{t}$ and $y_{i}=\frac{\lambda_{i}}{\sigma^{2}+\lambda_{i}}, \forall i=$ $1 \cdots N$ then (28) shows the relationship between $y_{1}, \cdots, y_{N}$ and $\beta_{1}, \cdots, \beta_{K}$. Now

$$
\begin{aligned}
\sum_{i=1}^{K} p_{i} & =\operatorname{tr}\left[S D S^{t}\right] \quad \text { see (17) } \\
& =\sigma^{2} \sum_{i=1}^{N} \frac{y_{i}}{1-y_{i}}
\end{aligned}
$$

The lemma below identifies a lower bound on $\sum_{i=1}^{K} p_{i}$. Recall that the set of oversized users is denoted by $\mathcal{K}$.

Lemma 5.1: Let $\mathcal{K}$ be the set of oversized users. Given the constraint (28)

$$
\min \left\{\sum_{i=1}^{N} \frac{y_{i}}{1-y_{i}}\right\}=\sum_{i \in \mathcal{K}} \beta_{i}+\frac{(N-|\mathcal{K}|) \sum_{j \not \mathcal{K}} e\left(\beta_{j}\right)}{N-|\mathcal{K}|-\sum_{j \notin \mathcal{K}} e\left(\beta_{j}\right)}
$$

Suppose that this is true. Then for any allocation of sequences and powers so that the SIR requirements of the users are met, (33) can be used in conjunction with (32) to obtain a lower bound on the sum of allocated received powers

$$
\sum_{i=1}^{K} p_{i} \geq \sigma^{2}\left(\sum_{i \in \mathcal{K}} \beta_{i}+\frac{(N-|\mathcal{K}|) \sum_{j \not \mathcal{K}} e\left(\beta_{j}\right)}{N-|\mathcal{K}|-\sum_{j \notin \mathcal{K}} e\left(\beta_{j}\right)}\right) .
$$

It is now straightforward to verify that the sum of powers allocated in the sufficiency proof of Theorem 5.1 (in (22)) meets this lower bound. Thus our specific valid allocation of sequences and powers is optimal in the above sense of minimizing the sum of allocated powers. We now prove the lemma.

Proof of Lemma 5.1: Let us develop some notation by defining the set over which $y_{1}, \cdots, y_{N}$ vary. Rewriting (28), let

$$
\begin{aligned}
\mathcal{N}=\left\{\left(y_{1}, \cdots, y_{N}\right) \in \mathcal{R}_{+}^{N}:\left(y_{1}, \cdots, y_{N}, 0, \cdots, 0\right)\right. \text { majorizes } \\
\\
\left.\left(e\left(\beta_{1}\right), \cdots, e\left(\beta_{K}\right)\right)\right\} .
\end{aligned}
$$

Observe that the map $x \mapsto \frac{x}{1-x}$ is convex and hence the symmetric convex map

$$
f(y)=\sum_{i=1}^{N} \frac{y_{i}}{1-y_{i}}
$$

is Schur-convex (see Definition 4.1 and Example 4.1). Hence if $y$ majorizes $\tilde{y}$ then $f(y)>f(\tilde{y})$. We now complete the proof of Lemma 5.1 by identifying a "Schur-minimal" element in $\mathcal{N}$. Let the set of oversized users be denoted by $\mathcal{K}$. Let $y^{*}=\left(y_{1}^{*}, \cdots, y_{N}^{*}\right) \in \mathbb{R}_{+}^{N}$ be

$$
y^{*}=\left(\frac{\sum_{j \notin \mathcal{K}} e\left(\beta_{j}\right)}{N-|\mathcal{K}|}, \cdots, \frac{\sum_{j \notin \mathcal{K}} e\left(\beta_{j}\right)}{N-|\mathcal{K}|}, e\left(\beta_{i}\right) ; i \in \mathcal{K}\right) \text {. }
$$

Consider the following claims:

$$
\begin{gathered}
y^{*} \in \mathcal{N} \\
y \text { majorizes } y^{*}, \quad \forall y \in \mathcal{N}
\end{gathered}
$$

Suppose these are true. Then $f\left(y^{*}\right)$ is the minimum value of the minimization problem in (33) and the claim in Lemma 5.1 is now straightforward to verify. We only need to prove (36) and (37) above.

It is straightforward to verify from the definition of $y^{*}$ and by properties of oversized users that $y^{*} \in \mathcal{N}$. Let $y=\left(y_{1}, \cdots, y_{N}\right) \in \mathcal{N}$ and $y_{[1]}, \cdots y_{[N]}$ denote the order statistics of $y$ (see Definition 3.1 for the notation). Let $\beta_{[1]}, \cdots, \beta_{[K]}$ be the order statistics of $\beta_{1}, \cdots, \beta_{K}$. By the definition of $y^{*}$ in (35) and properties of oversized users, it can be verified that the following relation is true among the elements of $y^{*}$ :

$$
\begin{aligned}
y_{[1]}^{*}=\max & \left\{\frac{\sum_{i=1}^{K} e\left(\beta_{i}\right)}{N}, e\left(\beta_{[1]}\right)\right\} \\
y_{[k+1]}^{*}=\max & \left\{\frac{\sum_{i=1}^{K} e\left(\beta_{i}\right)-\sum_{i=1}^{k} y_{[i]}^{*}}{N-k}, e\left(\beta_{[k+1]}\right)\right. \\
& \left.+\sum_{i=1}^{k}\left(e\left(\beta_{[i]}\right)-y_{[i]}^{*}\right)\right\}, \quad \forall k=1 \cdots N-1 .
\end{aligned}
$$

Hence $\forall k=1 \cdots N-1$ we can write

$$
\begin{aligned}
& \sum_{i=1}^{k+1} y_{[i]}^{*} \\
& =\max \left\{\sum_{i=1}^{k+1} e\left(\beta_{[i]}\right), \frac{\sum_{j=1}^{K} e\left(\beta_{j}\right)}{N-k}+\frac{N-k-1}{N-k} \sum_{i=1}^{k} y_{[i]}^{*}\right\} .
\end{aligned}
$$

Now, since $y \in \mathcal{N}$ we have

$$
\sum_{i=1}^{N} y_{i}=\sum_{i=1}^{K} e\left(\beta_{i}\right)
$$

and hence

$$
y_{[1]} \geq \frac{\sum_{i=1}^{K} e\left(\beta_{i}\right)}{N}
$$

Furthermore, $y_{[1]} \geq e\left(\beta_{[1]}\right)$. Hence

$$
y_{[1]} \geq \max \left\{\frac{\sum_{i=1}^{K} e\left(\beta_{i}\right)}{N}, e\left(\beta_{[1]}\right)\right\}=y_{[1]}^{*} .
$$

We complete the proof of the claim that $y$ majorizes $y^{*}$ by induction. Suppose

$$
\sum_{i=1}^{k} y_{[i]} \geq \sum_{i=1}^{k} y_{[i]}^{*}
$$


for some $1 \leq k<N$. Since

$$
\text { ' } \sum_{i=1}^{N-k} y_{[k+i]}=\sum_{j=1}^{K} e\left(\beta_{j}\right)-\sum_{i=1}^{k} y_{[i]}
$$

and $y_{[k+1]} \geq y_{[k+2]} \geq \cdots \geq y_{[N]}$, we have

$$
y_{[k+1]} \geq \frac{\sum_{j=1}^{K} e\left(\beta_{j}\right)-\sum_{i=1}^{k} y_{[i]}}{N-k} .
$$

Hence

$$
\begin{aligned}
\sum_{i=1}^{k+1} y_{[i]} & \geq \frac{\sum_{i=1}^{K} e\left(\beta_{i}\right)}{N-k}+\left(\frac{N-k-1}{N-k}\right) \sum_{i=1}^{k} y_{[i]} \\
& \geq \frac{\sum_{i=1}^{K} e\left(\beta_{i}\right)}{N-k}+\left(\frac{N-k-1}{N-k}\right) \sum_{i=1}^{k} y_{[i]}^{*}
\end{aligned}
$$

by induction hypothesis.

Since $\sum_{i=1}^{k+1}\left(y_{[i]}-e\left(\beta_{[i]}\right)\right) \geq 0$, from (40), we have

$$
\begin{aligned}
\sum_{i=1}^{k+1} y_{[i]} & \geq \max \left\{\sum_{i=1}^{k+1} e\left(\beta_{[i]}\right), \frac{\sum_{j=1}^{K} e\left(\beta_{j}\right)}{N-k}+\frac{N-k-1}{N-k} \sum_{i=1}^{k} y_{[i]}^{*}\right\} \\
& =\sum_{i=1}^{k+1} y_{[i]}^{*} \text { from (39). }
\end{aligned}
$$

This is true for all $k=1 \cdots N-1$. Hence $y$ majorizes $y^{*}$ and $y^{*}$ is a Schur-minimal element of $\mathcal{N}$.

\section{DOWNLINK AND USER CAPACITY}

Until now, we have been considering the uplink of the cellular system. In the downlink of this system there is a single transmitter (the base station) and there are multiple receivers (the users). We first formally define our model and then consider the user capacity of the downlink.

\section{A. Definitions and Model}

Suppose there are $K$ users in the downlink of the system. Let the path gain from the base station (interchangeably referred to as transmitter) to user $i$ be $h_{i}$. We suppose that the noise at the receivers is additive white Gaussian with the same variance $\sigma^{2}$ per degree of freedom for each user (there is no loss of generality in this assumption since we can incorporate this into the path gain parameter $h_{i}$ ). We say that $K$ users (with path gains from the base station being $h_{1}, \cdots, h_{K}$ and each having the same SIR requirement of $\beta$ ) are admissible in the downlink of the system with processing gain $N$ if we can allot transmit power $p_{i}$ and signature sequence $s_{i}$ at the transmitter corresponding to user $i$

$$
\operatorname{SIR}_{i}=\frac{p_{i} h_{i}\left(s_{i}, c_{i}\right)^{2}}{\sigma^{2}\left(c_{i}, c_{i}\right)+\sum_{j \neq i} p_{j} h_{i}\left(s_{j}, c_{i}\right)^{2}} \geq \beta
$$

where $c_{i}$ is the MMSE linear receiver given the signature sequences and the powers. Proceeding as in Section II-A, it is easy to verify that the optimal (in the sense of maximizing SIR for each user) linear receiver $c_{i}$ for user $i$ is

$$
c_{i}=\tilde{Z}_{i}^{-1} s_{i}
$$

where $\tilde{Z}_{i}=\frac{\sigma^{2}}{h_{i}} I+\sum_{j \neq i} p_{j} s_{j} s_{j}^{t}$ and the corresponding $\operatorname{SIR}_{i}$ with the optimal receiver is

$$
\mathrm{SIR}_{i}=s_{i}^{t} \tilde{Z}_{i}^{-1} s_{i} p_{i} .
$$

It is clear that we can make a similar definition of admissibility of the users when the receiver structure is fixed to be the matched filter. The similarity of the achieved SIR equation (41) to the corresponding one in the uplink in (2) is apparent. Only the noise variance $\sigma^{2}$ in (2) is replaced now by $\frac{\sigma^{2}}{h_{i}}$. If we have no constraints on the allocated power we can "null out" the additive noise and the admissibility characterization is identical to that of the uplink.

\section{B. User Capacity Characterization}

Theorem 6.1: $K$ users with path gains $h_{1}, \cdots, h_{K}$ from the base station and each having the same SIR requirement $\beta$ are admissible in the downlink of the system with processing gain $N$ if and only if

$$
K<N\left(1+\frac{1}{\beta}\right) .
$$

Proof: We first show the necessity, on the same lines of the uplink situation. Suppose the $K$ users are admissible in the downlink. Then, for each user $i$, there exists signature sequence $s_{i}$ and transmit power at the base station $p_{i}$ (as a function of the path gains $\left.h_{1}, \cdots, h_{K}\right)$ such that the achieved SIR of user $i$ (as in (41)) is greater than or equal to $\beta$. Using (43)

$$
s_{i}^{t} \tilde{Z}_{i}^{-1} s_{i} p_{i} \geq \beta \text {. }
$$

Proceeding as in (5), we have, for each user $i$

$$
s_{i}^{t} \tilde{Z}_{i}^{-1} s_{i} p_{i}=\frac{s_{i}^{t} \hat{Z}_{i}^{-1} s_{i} p_{i}}{1-s_{i}^{t} \hat{Z}_{i}^{-1} s_{i} p_{i}}
$$

where $\hat{Z}_{i}=\tilde{Z}_{i}+p_{i} s_{i} s_{i}^{t}$. Recalling the notation developed in Section II, $S=\left[s_{1}, \cdots, s_{K}\right]$ and $D=\operatorname{diag}\left\{p_{1}, \cdots, p_{K}\right\}$ and $S D S^{t}=U \Lambda U$. Then we can rewrite $\hat{Z}_{i}=\frac{\sigma^{2}}{h_{i}} I+S D S^{t}$. We have, from (44), that for each user $i$,

$$
s_{i}^{t} \hat{Z}_{i}^{-1} s_{i} p_{i} \geq \frac{\beta}{1+\beta}
$$

Equivalently, we have for each user $i$

$$
\left(U s_{i}\right)^{t}\left(\frac{\sigma^{2}}{h_{i}} I+\Lambda\right)^{-1}\left(U s_{i}\right) p_{i} \geq \frac{\beta}{1+\beta} .
$$

Denoting $\hat{h}=\max _{i=1}^{K} h_{i}$, we have for each user $i$

$$
\begin{aligned}
\frac{\beta}{1+\beta} & \leq\left(U s_{i}\right)^{t}\left(\frac{\sigma^{2}}{\hat{h}} I+\Lambda\right)^{-1}\left(U s_{i}\right) p_{i} \\
& =s_{i}^{t}\left(\frac{\sigma^{2}}{\hat{h}} I+S D S^{t}\right)^{-1} s_{i} p_{i} .
\end{aligned}
$$

Summing up the terms, we have

$$
\begin{aligned}
\frac{K \beta}{1+\beta} & \leq \operatorname{tr}\left[S^{t}\left(\frac{\sigma^{2}}{\hat{h}} I+S D S^{t}\right)^{-1} S D\right] \\
& =\operatorname{tr}\left[S D S^{t}\left(\frac{\sigma^{2}}{\hat{h}} I+S D S^{t}\right)^{-1}\right] \\
& <N .
\end{aligned}
$$


To show this is sufficient as well, suppose $K<N\left(1+\frac{1}{\beta}\right)$ and $h_{1}, \cdots, h_{K}$ be arbitrary positive real numbers. Allot WBE signature sequences for the users and powers

$$
p_{i}=p=\frac{\sigma^{2}}{\breve{h}\left(1+\frac{1}{\beta}-\frac{K}{N}\right)}, \quad \forall i=1 \cdots K
$$

where $\breve{h}=\min _{i=1}^{K} h_{i}$. Then, $S S^{t}=\frac{K}{N} I$ and hence for every user $i$ we have

$$
\hat{Z}_{i}=\left(\frac{\sigma^{2}}{h_{i}}+\frac{K p}{N}\right) I .
$$

Using (45), $\forall i=1 \cdots, K$

$$
\begin{aligned}
\operatorname{SIR}_{i} & =\frac{p\left(\frac{\sigma^{2}}{h_{i}}+\frac{K p}{N}\right)^{-1}}{1-p\left(\frac{\sigma^{2}}{h_{i}}+\frac{K p}{N}\right)^{-1}} \\
& =\frac{p}{\frac{\sigma^{2}}{h_{i}}+\frac{K p}{N}-p} \\
& =\frac{\beta}{\frac{\check{h}}{h_{i}}\left(1+\beta-\frac{K \beta}{N}\right)+\frac{K \beta}{N}-\beta} \\
& \geq \beta
\end{aligned}
$$

where we used the fact that

$$
\frac{\breve{h}}{h_{i}}\left(1+\beta-\frac{K \beta}{N}\right)+\frac{K \beta}{N}-\beta \leq 1
$$

since by hypothesis we have that $K<N\left(1+\frac{1}{\beta}\right)$ and $\breve{h} \leq h_{i}$. Hence the $K$ users are admissible.

Some comments are in order now:

1) A (simple) closed-form expression of the allocation that is optimal (in the sense of the previous sections) seems unattainable. But it is worth emphasizing that our allocation above has the property that the signature sequences are allocated (to be WBE sequences) independent of the path gains and only the powers are chosen as a function of the path gain.

2) With matched-filter receivers, the admissibility is unchanged and we have $K$ users with path gains $h_{1}, \cdots, h_{K}$ from the base station and each having the same SIR requirement $\beta$ are admissible in the downlink of the system with processing gain $N$ if and only if

$$
K<N\left(1+\frac{1}{\beta}\right) \text {. }
$$

3) When users have different SIR requirements, a simple calculation shows that $K$ users with path gains from the base station $h_{1}, \cdots, h_{K}$ and having SIR requirements $\beta_{1}, \cdots, \beta_{K}$ are admissible in the downlink of the system with processing gain $N$ if and only if

$$
\sum_{i=1}^{K} \frac{\beta_{i}}{1+\beta_{i}}<N
$$

The same statement is true when using matched-filter receivers instead of MMSE receivers.

4) For every class $l, \alpha_{l}$ users per unit processing gain of that class are admissible by "channelizing" the downlink of a large enough system if

$$
\sum_{l=1}^{N} \frac{\alpha_{l} \beta_{l}}{1+\beta_{l}}<1 .
$$

\section{JOINT FDMA/CDMA CASE AND USER CAPACITY}

Traditional multiple-access schemes divide the channel into slots and it is important to note that we can incorporate a slotted system into our framework. We achieve this by forcing the signature sequences to be chosen only from an orthogonal sequence set. Then users that have the same signature sequence are in the same "slot" or "channel" and do not cause any interference to users in different "channels" due to the orthogonality of the signature sequences. In this case, the receiver is trivial and the MMSE and matched-filter receivers coincide. It is interesting to identify the user capacity in this situation and this exercise will enable us to explicitly identify the gain in user capacity by using nonorthogonal signature sequences. In this section, we identify the user capacity of the slotted system in a variety of settings. The conclusion we draw from the results is that the user capacity in this case differs from the earlier one by an integer part of a function of the SIR requirement. The assumption below is that the signature sequences are now constrained to be chosen from an orthogonal sequence set (whose linear span has dimension equal to the processing gain of the system). We first focus on the uplink.

Proposition 7.1: $K$ users each with SIR requirement $\beta$ are admissible in the system having processing gain $N$ if and only if

$$
\begin{array}{ll}
K<N\left(1+\frac{1}{\beta}\right), & \text { if }\left\lfloor\frac{1}{\beta}\right\rfloor=\frac{1}{\beta} \\
K \leq N\left\lfloor 1+\frac{1}{\beta}\right\rfloor, & \text { else. }
\end{array}
$$

Proof: Since the sequences are chosen from an orthogonal set, only users having the same sequence (we shall refer to them as users in the same channel) cause interference to each other. We hence focus on the user capacity for a single channel. $\tilde{K}$ users are admissible into a channel with SIR requirement $\beta$ if there exist positive powers $p_{1}, \cdots, p_{\tilde{K}}$ such that, analogous to $(2)$

$$
\operatorname{SIR}_{i}=\frac{p_{i}}{\sigma^{2}+\sum_{j \neq i} p_{j}} \geq \beta, \quad \forall i=1 \cdots \tilde{K} .
$$

The existence of such powers can be seen to be equivalent to (see [16, Theorem 2.1])

$$
r(A)<1+\frac{1}{\beta}
$$

where $r(\cdot)$ is the Perron-Frobenius eigenvalue of the argument (which is a nonnegative irreducible matrix; for notation and definition see [16, Ch. 1]) and $A$ is a $\tilde{K} \times \tilde{K}$ matrix with all entries being equal to 1 . Since $r(A)=\tilde{K}$, the existence of powers satisfying (49) is equivalent to the number of users $\tilde{K}<1+\frac{1}{\beta}$. Since we have $N$ channels available, this is equivalent to the total number of users $K<N\left(1+\frac{1}{\beta}\right)$ if $\left\lfloor\frac{1}{\beta}\right\rfloor=\frac{1}{\beta}$ and $K \leq N\left\lfloor 1+\frac{1}{\beta}\right\rfloor$ else. 
In the general situation when users have arbitrary SIR requirements, we summarize our results without detailing the proofs.

1) We focus on a single channel first. $K$ users with SIR requirements $\beta_{1}, \cdots, \beta_{K}$ are admissible in a single channel if and only if

$$
\sum_{i=1}^{K} \frac{\beta_{i}}{1+\beta_{i}}<1 .
$$

2) When there are $N$ channels, a sufficient condition for admissibility of $K$ users with SIR requirements $\beta_{1}, \cdots, \beta_{K}$ is

$$
\sum_{i=1}^{K} \frac{\beta_{i}}{1+\beta_{i}}<N .
$$

A necessary and sufficient condition for admissibility is that there be a way to allot every user to one (among $N$ ) channel such that users within each channel are admissible.

Proposition 7.2: $K$ users each with SIR requirement $\beta$ are admissible in the system having processing gain $N$ and power constraint $P$ if and only if

$$
K \leq N\left\lfloor 1+\frac{1}{\beta}-\frac{\sigma^{2}}{\bar{P}}\right\rfloor
$$

Let us consider the single channel first. $K$ users are admissible in the single channel if there exist positive powers $p_{1}, \cdots, p_{K}$ each upper-bounded by $\bar{P}$ such that for each user $i$

$$
\operatorname{SIR}_{i}=\frac{p_{i}}{\sigma^{2}+\sum_{j \neq i} p_{j}} \geq \beta
$$

We can rewrite this in matrix notation as

$$
\left(I-\frac{\beta e e^{t}}{1+\beta}\right) p \geq \frac{\beta \sigma^{2}}{1+\beta} e
$$

where $e$ is a $K \times 1$ vectors of all ones. As in (50), the existence of such positive powers is seen to be equivalent to $r\left(e e^{t}\right)=K<1+\frac{1}{\beta}$. Furthermore, under this condition, there is a component-wise minimal power solution, (see [16, Theorem 2.1]) given by

$$
\begin{aligned}
\bar{p} & =\frac{\beta \sigma^{2}}{1+\beta}\left(I-\frac{\beta e e^{t}}{1+\beta}\right)^{-1} e \\
& =\frac{\beta \sigma^{2}}{1+\beta-K \beta} e \quad \text { after some elementary algebra. }
\end{aligned}
$$

Thus $K$ users are admissible in the single channel with power constraint $P$ if and only if $K<1+\frac{1}{\beta}$ and $\frac{\beta \sigma^{2}}{1+\beta-K \beta} \leq \bar{P}$. This is equivalent to

$$
K \leq 1+\frac{1}{\beta}-\frac{\sigma^{2}}{\bar{P}}
$$

Since there are $N$ channels, we conclude that this is equivalent to the total number of users (in all the $N$ channels) $K \leq$ $N\left\lfloor 1+\frac{1}{\beta}-\frac{\sigma^{2}}{\bar{P}}\right\rfloor$.

As was done earlier, we state the user capacity for the downlink system in this situation without detailing the proofs.
The proofs can be obtained by an argument similar to the ones made above.

1) $K$ users (with path gains from the base station being $h_{1}, \cdots, h_{K}$ ) each with SIR requirement $\beta$ are admissible in the downlink of the system having processing gain $N$ if and only if

$$
\begin{array}{ll}
K<N\left(1+\frac{1}{\beta}\right), & \text { if }\left\lfloor\frac{1}{\beta}\right\rfloor=\frac{1}{\beta} \\
K \leq N\left\lfloor 1+\frac{1}{\beta}\right\rfloor, & \text { else. }
\end{array}
$$

2) $K$ users (with path gains from the base station being $\left.h_{1}, \cdots, h_{K}\right)$ with SIR requirements $\beta_{1}, \cdots, \beta_{K}$ are admissible in a single channel in the downlink if and only if

$$
\sum_{i=1}^{K} \frac{\beta_{i}}{1+\beta_{i}}<1
$$

\section{CONCLUSIONS AND Future WORK}

Most previous studies of multiuser receivers (see [22] for a comprehensive study) are user-centric. Specifically, typical measures such as near-far resistance focus on the performance of a single user in the face of worst case interference. Different from these works, here, we consider a network-centric formulation where the users have to simultaneously satisfy their performance requirements and network-level user capacity is the ultimate performance measure. Despite the simplistic setting of symbol synchronism and no fading (equivalently, perfect channel estimation and power control) this formulation allows us to study the fundamental tradeoffs between the performance of different users through the allocation of signature sequences and power control. Though our model does not make any restrictions on the users' symbols $X_{i}$, we have a heavily coded system (such as that of IS-95) in mind and that the MMSE estimates are used for soft decoding of the users' raw bits. The desired $\frac{E_{s}}{I_{0}}$ for IS-95 system is about 3-7 dB (see [24, p. 183]) and this corresponds to an SIR requirement of about $\beta=3$.

The huge literature on design of signature sequences for various communication models is comprehensively covered in [3]. The assumption of perfect power control made in this paper is relaxed and results here are extended in [1]. Motivated by the optimal nature of WBE sequences, in [19], the authors provide an iterative interference suppression algorithm that updates the signature sequences allocated to the users and demonstrate that the allocations converge to WBE sequences.

\section{APPENDIX \\ EXISTENCE AND CONSTRUCTION OF GENERALIZED WBE SEQUENCES}

We have identified generalized WBE sequences as the optimal sequence allocation in Section V. In the proof of Theorem 5.1, we also illustrated a procedure to construct these sequences. In this appendix we discuss some characterizations of these sequences and mention some open problems. As a reprise, we repeat the definition of generalized WBE sequences: Fix $K \geq N$ henceforth. Fix $D$, the diagonal 
matrix of user powers $p_{1}, \cdots, p_{K}$. Let $p_{\text {tot }}=\sum_{i=1}^{K} p_{i}$. Then, say that $s_{1}, \cdots, s_{K} \in \mathcal{S}_{1}^{N-1}$ (the unit sphere in $\mathbb{R}^{N}$ ) are generalized WBE sequences and the matrix $S=\left[s_{1}, \cdots, s_{K}\right]$ as a generalized WBE matrix if the following three conditions are satisfied:

1) The rows of $S$ have the same $l_{2}$ norm, equal to $\sqrt{\frac{p_{\text {tot }}}{N}}$.

2) The columns of $S$ have unit $l_{2}$ norm.

3) The rows of $S D^{\frac{1}{2}}$ are orthogonal to each other.

Properties 1) and 3) can also be succinctly expressed as $S D S^{t}=\frac{p_{\text {tot }}}{N} I$. When $K=N$ and $D$ is scaled identity, orthonormal matrices are the only matrices satisfying the above three properties. When $K=N$ and $D$ is not the scaled identity, then there is no generalized WBE matrix. We now generalize this observation to arbitrary $K>N$.

The matrix $D^{\frac{1}{2}} S^{t} S D^{\frac{1}{2}}$ has the same eigenvalues as $S D S^{t}$ along with $N$ additional zero eigenvalues. Since the diagonal entries of $D^{\frac{1}{2}} S^{t} S D^{\frac{1}{2}}$ are $p_{1}, \cdots, p_{M}$, a necessary condition for the existence of a generalized WBE matrix $S$ is, referring to Lemma 3.1

$$
\begin{aligned}
& \left(\frac{p_{\mathrm{tot}}}{N}, \cdots, \frac{p_{\mathrm{tot}}}{N}, 0, \cdots, 0\right) \text { majorizes }\left(p_{1}, \cdots, p_{K}\right) \\
& \quad \text { which simplifies to } \frac{p_{\mathrm{tot}}}{N} \geq p_{i}, \quad \forall i=1, \cdots, K
\end{aligned}
$$

Observe that when $K=N$, (51) reduces to the condition that $p_{1}=p_{2}=\cdots=p_{K}$. As suggested by Lemma 3.1, this condition is also sufficient for the existence of a generalized WBE matrix $S$. To see this, suppose (51) holds. Then, by Lemma 3.1, there exists a real symmetric matrix, say $P$, with diagonal entries $p_{1}, \cdots, p_{K}$ and eigenvalues $\frac{p_{\text {tot }}}{N}$ and 0 with multiplicities (both geometric and algebraic) $N$ and $K-N$, respectively. Let us denote the set of such matrices by $\mathcal{P}$ and $P$ as an element of this set. Also, let $v_{1}, \cdots, v_{L}$ be the normalized eigenvectors of $P$ corresponding to the eigenvalue $\frac{p_{\text {tot }}}{N}$ (written as elements of $\mathbb{R}^{1 \times K}$ ). We claim that the definition

$$
S=\sqrt{\frac{p_{\text {tot }}}{N}}\left[\begin{array}{c}
v_{1} \\
v_{2} \\
\vdots \\
v_{N}
\end{array}\right] D^{-\frac{1}{2}}
$$

satisfies the three properties of generalized WBE matrix. Properties 1) and 3) are satisfied by definition. It is trivial to verify that $S^{t} S$ has unit diagonal entries and hence property 2 ) is satisfied also. The important observation is that every generalized WBE matrix is generated in this way: If $S$ is a generalized WBE matrix, then the $N$ rows of $V \triangleq \sqrt{\frac{N}{p_{\text {tot }}}} S D^{\frac{1}{2}}$ serves as the eigenvectors corresponding to the eigenvalue $\frac{p_{\text {tot }}}{N}$ of some matrix $P \in \mathcal{P}$. Also, it is a trivial observation that, if $S$ is a generalized WBE matrix, then so is $Q S$ for any orthonormal matrix $Q$, i.e., rotating all the generalized WBE sequences by the same rotation matrix does not alter their properties.

Given their optimality, it is important to characterize generalized WBE matrices up to an equivalence class of orthonormal rotations. Such a characterization will aid design questions such as constructing sequences with entries constrained to be in $\{+1,-1\}$ or with a peak power constraint on the entries. Though we have characterized all generalized WBE matrices through the eigenvectors of certain positive-definite matrices with fixed diagonal entries and fixed eigenvalues, this characterization does not seem to aid, in a straightforward way, the answering of the design questions mentioned above. Nevertheless, this has afforded us some intuition on constructing generalized WBE matrices; in [23], we demonstrate a constructive (iterative, with at most $K$ iterations) algorithm to construct the eigenvectors $v_{1}, \cdots, v_{N}$ of the matrix $P \in \mathcal{P}$.

For the special case when $D$ is the identity, the matrix $S$ reduces to a WBE matrix and simple construction schemes are known.

1) WBE matrix goes by the name of tight frame ${ }^{2}$ in the context of over complete expansions in $\mathbb{R}^{N}$ in Wavelets literature (see [2, Ch. 3] for a detailed review of tight frames). The following construction of tight frames is well known: assuming $N$ is odd, the $k$ th sequence is given by

$$
\begin{aligned}
\sqrt{\frac{2}{N}}\left(\frac{1}{\sqrt{2}}, \cos \right. & \frac{2 \pi k}{K}, \sin \frac{2 \pi k}{K}, \cdots, \\
& \left.\cos \frac{2 \pi(N-1) k}{2 K}, \sin \frac{2 \pi(N-1) k}{2 K}\right)
\end{aligned}
$$

for $1 \leq k \leq K$. When $N$ is even, we construct $K$ sequences as above (replacing $N$ by $N+1$ ) and ignore the first element (namely, $\frac{1}{\sqrt{2}}$ ) of each sequence and scale the resulting sequence by

$$
\sqrt{\frac{N+1}{N}}
$$

to normalize it. It is easily verified that this constructs WBE sequences for arbitrary $K \geq N$.

2) In [12], the authors construct WBE sequences with entries restricted to $\{+1,-1\}$ when $K=2^{l}$ for $l \leq N$.

3) We remark that constructing WBE sequences for every $K \geq N$ with entries restricted to $\{+1,-1\}$ is equivalent to solving the long-standing open problem of constructing Hadamard matrices for every dimension a multiple of 4 (see [5] for details and further references on this problem).

4) Recently, in [19], motivated by the demonstration of the optimal nature of WBE sequences, the authors have described a distributed algorithm that updates iteratively the signature sequences of the users. Given an initial set of signature sequences for the users (that has some very weak properties) the authors show that the users' signature sequences converge to WBE sequences using this iterative algorithm.

\section{REFERENCES}

[1] E. K. P. Chong and J. Zhang, "Admissibility and network capacity of power-controlled CDMA systems in fading channels," in 36th Annual Allerton Conf. Communication, Control and Computing, 1998.

\footnotetext{
${ }^{2}$ We would like to thank Vivek Goyal and Prof. Martin Vetterli for pointing this out to us.
} 
[2] I. Daubechies, Ten Lectures on Wavelets. Philadelphia, PA: SIAM, 1992.

[3] P. Fan and M. Darnell, Sequence Design for Communication Applications. New York: Wiley, 1996.

[4] G. J. Foschini and Z. Miljanic, "A simple distributed autonomous power control algorithm and its convergence" IEEE Trans. Vehic. Technol., vol. 40, no. 4. pp. 641-646, 1993.

[5] R. L. Graham, M. Grotschel, and L. Lovasz, Handbook of Combinatorics, vol. 2. Cambridge, MA: MIT Press, 1995.

[6] S. V. Hanly, "An algorithm for combined cell-site selection and power control to maximize cellular spread spectrum capacity" IEEE J. Select. Areas Commun. (Special Issue on the Fundamentals of Networking), vol. 13, no. 7, Sept. 1995.

[7] P. Kumar and J. Holtzman, "Power control for a spread-spectrum system with multiuser receivers," in Proc. 6th IEEE PIMRC, Sept. 1995, pp. 955-959.

[8] R. Lupas and S. Verdú, "Linear multiuser detectors for synchronous code-division multiple access," IEEE Trans. Inform. Theory, vol. 35, pp. 123-136, Jan. 1989.

[9] _ "Near-far resistance of multiuser detectors in asynchronous channels," IEEE Trans. Commun., vol. 38, pp. 496-508, Apr. 1990.

[10] U. Madhow and M. Honig, "MMSE interference suppression for directsequence spread-spectrum CDMA," IEEE Trans. Commun., vol. 42, pp. 3178-3188, Dec. 1994.

[11] A. W. Marshall and I. Olkin, Inequalities: Theory of Majorization and Its Applications. New York: Academic, 1979.

[12] J. L. Massey and T. Mittelholzer, "Welch's bound and sequence sets for code-division multiple access systems," in Sequences II, Methods in Communication, Security and Computer Science, R. Capocelli, A. De Santis, and U. Vaccaro, Eds. New York: Springer-Verlag, 1993.

[13] M. Rupf and J. L. Massey, "Optimum sequence multisets for synchronous code-division multiple-access channels," IEEE Trans. Inform. Theory, vol. 40, pp. 1261-1266, July 1994.
[14] P. Rapajic and B. Vucetic, "Adaptive receiver structures for asynchronous CDMA systems," IEEE J. Select. Areas Commun., vol. 12, pp. 685-697, May 1994.

[15] M. Rupf, F. Tarköy, and J. Massey, "User-separating demodulation for code-division multiple access systems," IEEE J. Select. Areas Commun., vol. 12, pp. 786-795, June 1994.

[16] E. Seneta, Nonnegative Matrices and Markov Chains, 2nd ed. Berlin, Germany: Springer-Verlag, 1981.

[17] D. Tse and S. Hanly, "Multiuser demodulation: Effective interference, effective bandwidth and capacity," IEEE Trans. Inform. Theory, vol. 45, pp. 641-657, Mar. 1999

[18] S. Ulukus and R. Yates, "Adaptive power control and MMSE interference suppression," ACM Wireless Networks, vol. 4, no. 6, pp. 489-496, 1998.

[19] _ "Iterative signature adaptation for capacity maximization of CDMA systems," in 36th Annual Allerton Conf. Communication, Control and Computing, 1998.

[20] M. K. Varanasi and T. Guess, "Optimum decision feedback multiuser equalization and successive decoding achieves the total capacity of the Gaussian multiple-access channel," in Proc. Asilomar Conf. Signals, Systems and Computers, 1997.

[21] S. Verdú, "Optimum multiuser asymptotic efficiency," IEEE Trans. Commun., vol. 34, pp. 890-897, Sept. 1996.

[22] _ Multiuser Detection. Cambridge, U.K.: Cambridge Univ. Press, 1998.

[23] P. Viswanath and V. Anantharam, "Optimal sequences and sum capacity of synchronous CDMA systems," this issue, pp. 1984-1991.

[24] A. J. Viterbi, CDMA-Principles of Spread Spectrum Communication. Reading, MA: Addison-Wesley, 1995.

[25] L. R. Welch, "Lower bounds on the maximum cross correlation of signals," IEEE Trans. Inform. Theory, vol. IT-20, pp. 397-399, May 1974.

[26] Z. Xie, Short, and C. Rushforth, "A family of suboptimum detectors for coherent multiuser communications," IEEE J. Select. Areas Commn., vol. 8, pp. 683-690, May 1990. 